\title{
Réfrigération solaire à adsorption solide : choix du meilleur couple d'adsorption
}

\author{
A. Adell (*) \\ Département de physique atmosphérique, Université d'Abidjan, 04 BP 322, Abidjan, Côte d'Ivoire
}

(Reçu le 6 juin 1983, révisé le 21 octobre 1983 et le 25 juin 1984, accepté le 19 septembre 1984)

Résumé. - Il a été recherché une formulation analytique approchée du coefficient de performance des réfrigérateurs solaires à adsorption solide, en fonction des caractéristiques physico-chimiques du couple d'adsorption et des conditions d'utilisation. Cette formule facilitera la recherche des meilleurs couples permettant de satisfaire un problème frigorifique donné dans des conditions optima. Un exemple de discussion a été fait entre ies couples zéolithe $13 \mathrm{x}$-eau et gel de silice-eau.

Abstract. - An analytical formula of solid adsorption solar refrigerators C.O.P., has been given in function of physical and chemical characteristics of the adsorption couple and the conditions of utilization. This formulation will facilitate the research of the best couples which allow to satisfy a given frigorific problem, in an optimum way. An example of discussion between zeolite $13 \mathrm{x}$-water and silica gel-water, has been done.

\section{Notations.}

\begin{tabular}{|c|c|}
\hline $\mathrm{maz}$ & $\begin{array}{l}\text { : température maximum atteinte par le } \\
\text { capteur à la désorption. }\end{array}$ \\
\hline $\mathrm{sec}$ & $\begin{array}{l}\text { : température à laquelle peut commencer } \\
\text { la désorption. }\end{array}$ \\
\hline . & $\begin{array}{l}\text { : température atteinte par le capteur en fin } \\
\text { d'adsorption. }\end{array}$ \\
\hline & $\begin{array}{l}\text { : température limite du capteur au-delà de } \\
\text { laquelle plus aucune désorption ne se } \\
\text { produit. }\end{array}$ \\
\hline & $\begin{array}{l}\text { : température maximum accessible par le } \\
\text { capteur pour une irradiation donnée. }\end{array}$ \\
\hline & $\begin{array}{l}\text { : température du capteur qui optimise le } \\
\text { rendement exergétique du système (cha- } \\
\text { leur utile et chaleur sensible). }\end{array}$ \\
\hline & $\begin{array}{l}\text { : température de l'air ambiant (état de } \\
\text { référence de l'exergie). }\end{array}$ \\
\hline & : température du condenseur. \\
\hline & $\begin{array}{l}\text { température minimale accessible à l'éva- } \\
\text { porateur. }\end{array}$ \\
\hline & $\begin{array}{l}\text { : température de désorption de la } i \text {-ième } \\
\text { tranche de fluide frigorigène. }\end{array}$ \\
\hline
\end{tabular}

(*) Nouvelle adresse : Laboratoire de chimie-physique, Université des Sciences et Techniques du Languedoc, place E. Bataillon, 34000 Montpellier, France.
$m(T, P) \quad$ : masse de fluide adsorbée à la température $T$ et à la pression $P$.

$\overline{\Delta m} \quad:$ tranche de fluide frigorigène.

$m_{0} \quad$ : masse maximum de fluide adsorbé par l'adsorbant.

A : coefficient d'adsorption caractéristique du couple.

$Q_{\text {c }} \quad:$ chaleur fournie par le capteur.

$Q_{\perp}^{*} \quad$ : irradiation solaire globale normale au capteur.

$Q_{\text {s }} \quad:$ chaleur sensible utilisée dans l'échauffement du bâti, de l'adsorbant et du fluide adsorbé.

$Q_{\mathrm{u}} \quad:$ chaleur utile qui sert à actionner le cycle frigorifique.

$\eta_{\mathrm{EX}} \quad:$ rendement exergétique $=$

$$
=\frac{\text { exergie désirée }}{\text { exergie nécessaire }}
$$

$\eta_{\mathrm{EX}}^{\mathrm{c}}\left(T_{\max }\right):$ rendement exergétique journalier du capteur.

$\eta_{\mathrm{EX}}^{\text {syst }} \quad:$ rendement exergétique du système frigorifique (chaleur utile et chaleur sensible).

$C^{\prime} \quad$ : capacité thermique totale du capteur par kg d'adsorbant.

$C_{\mathrm{p}} \quad$ : capacité thermique massique (adsorbant, adsorbat, ...)

$\mu \quad$ : valeur en eau du bâti par kg d'adsorbant. 


\section{Introduction.}

Le principe de fonctionnement des réfrigérateurs solaires à adsorption solide a été décrit par Meunier [1] et Tchernev [2]. Ce type d'appareil encore peu connu, s'apparente aux appareils à pompe chimique à affinité, tels que les machines à absorption liquide, mais la présence d'un adsorbant microporeux à l'état solide, non transférable entre le générateur et l'absorbant, donne au fonctionnement son caractère intermittent. Le cycle subi par le fluide frigorigène se décompose en deux étapes : une régénération sous l'action du rayonnement solaire le jour, suivie d'une production de froid nocturne.

Plusieurs prototypes de petite taille, expérimentés avec différents couples d'adsorption aussi bien en laboratoire qu'in situ sous différents climats [3], ont démontré la faisabilité du procédé. Des appareils plus puissants, dont la surface de captation dépasse $10 \mathrm{~m}^{2}$, sont actuellement à l'essai.

Ce type d'appareil qui peut constituer une filière intéressante pour produire du froid à partir de l'énergie solaire dans les pays en développement, a pour inconvénient une capacité de réfrigération insuffisante, d'une part pour satisfaire certains types de besoin important tels la climatisation des locaux, et d'autre part pour amortir les investissements à bref délai. Une recherche des conditions optima de fonctionnement s'avère nécessaire. Pour cette recherche de l'optimisation, une des questions essentielles est la détermination du couple d'adsorption présentant les meilleurs critères d'utilisation.

Dans le présent travail, consacré à l'étude théorique du choix du meilleur couple d'adsorption, le seul critère retenu a été la recherche du maximum $\mathrm{du}$ rendement frigorifique de la machine. Le calcul fait, à partir de l'analyse des irréversibilités de la machine, a permis de trouver une formule analytique donnant le coefficient de performance en fonction d'un grand nombre de paramètres. Le résultat a été obtenu grâce à certaines approximations qui ont été discutées. Il doit permettre une comparaison approchée des performances théoriques à attendre de ce type d'appareil pour différents couples d'adsorption et dans une grande variété de situations.

\section{Généralités sur les problèmes d'optimisation des réfrigérateurs solaires.}

L'optimisation consiste à rechercher les meilleures conditions d'utilisation de l'appareil en vu de satisfaire certains critères préalablement établis. Les problèmes d'optimisation acquièrent une certaine complexité en réfrigération [4] et plus encore en réfrigération solaire [5].

On distingue trois types de critères d'optimisation :

- techniques

- thermodynamiques

- économiques.
Pour les critères thermodynamiques, trois catégories de problèmes de complexité croissante se posent : on peut avoir à optimiser le fonctionnement d'un élément constitutif de la machine, ou bien le fonctionnement d'ensemble de la machine, ou encore la gestion du froid en tenant compte des besoins et de la variabilité de l'irradiation solaire.

La recherche des critères d'optimisation purement thermodynamique à laquelle la présente étude est limitée, est d'une grande importance en réfrigération solaire pour deux raisons :

a) la capacité frigorifique des appareils les plus performants actuels, ne dépasse pas cinq cents frigories par $\mathrm{m}^{2}$ et par jour; si on veut pouvoir les utiliser avec une surface de captation limitée, il faut optimiser leur efficacité afin d'éviter les sources énergétiques d'appoint.

b) L'incidence économique est en général aisée à déterminer : tout gain d'efficacité permet de réduire la surface de captation et donc le coût du capteur dans les mêmes proportions.

\section{Rappels des propriétés des cycles intermittents de réfrigération solaire.}

Une des caractéristiques fondamentales des cycles intermittents de réfrigération, est qu'ils ne peuvent fonctionner que selon un régime variable, durant lequel les paramètres thermodynamiques qui caractérisent l'évolution du fluide frigorigène, varient notablement au cours de la journée. Il est alors difficile de déterminer avec précision les qualités thermodynamiques de la machine à partir des valeurs moyennes de ces paramètres - comme on le fait par exemple pour le régime établi périodique des machines à compression - compte tenu des variations importantes qu'ils subissent : la température du capteur varie par exemple de plus de $60^{\circ} \mathrm{C}$ au cours de la désorption. Il a été proposé [6] de décomposer la masse totale de fluide cyclé en un certain nombre de «tranches" $\overline{\Delta m}$; chacune de ces tranches correspond à un cycle qui est parcouru d'une façon continue et dans des conditions moyennes mieux précisées. Les paramètres physiques de chacun de ces cycles, et notamment les températures d'adsorption et de désorption, sont obtenus à partir de l'équation semi-empirique de Dubinin, prise sous forme simplifiée

$$
m(T, P)=m_{0} \exp -A\left(\frac{T-T_{0}}{T_{0}}\right)^{2}
$$

où $m(T, P)$ représente la masse de gaz adsorbée par l'adsorbant microporeux à la température $T$, et à la pression $P$ correspondant à une température de saturation du gaz de $T_{0}: m_{0}$ est la masse maximum de gaz adsorbable (condensation capillaire exclue) et $A$ est une constante caractéristique du couple d'adsorption. 
4. Optimisation thermodynamique des réfrigérateurs solaires à adsorption solide.

Le schéma des flux énergétiques à l'intérieur des réfrigérateurs solaires à adsorption, s'établit comme suit :

chaleur sensible $\left(Q_{\mathrm{s}}\right)$ (bâti, adE.S. $\left(Q_{\perp}^{*}\right) \stackrel{\text { Captation }}{\longrightarrow} \begin{gathered}\text { Système } \\ \text { frigorifique }\end{gathered}{ }^{\left(Q_{\mathrm{c}}\right)} \begin{aligned} & \begin{array}{l}\text { sorbant, fluide } \\ \text { adsorbé) } \\ \text { chaleur utile } \\ \text { pour actionner } \\ \text { le cycle }\left(Q_{\mathrm{u}}\right)\end{array} \\ & \text { le }\end{aligned}$

Le calcul du rendement global de l'appareil fait intervenir deux étapes : le calcul du rendement du capteur solaire et le calcul du rendement du système frigorifique. Le choix du couple d'adsorption influe directement sur le rendement frigorifique qui est donc le facteur essentiel permettant de faire le choix du meilleur couple. Cependant le rendement du capteur est également affecté par le choix du couple d'une manière indirecte par le biais de la température atteinte à l'intérieur du capteur par le couple au cours de la désorption. Toutefois, pour un couple donné, il existe un type de capteur " adapté » qui est tel que, dans les conditions de fonctionnement choisies, les rendements exergétiques du capteur et du système frigorifique soient ensemble optima.

Pour le calcul du rendement exergétique du système frigorifique, il a été considéré, comme première hypothèse simplificatrice, que la loi des variations des isobares de désorption est du type :

$$
m(T)=a T+b .
$$

Cette loi est assez bien vérifiée expérimentalement par l'équation de Dubinin (1), au voisinage du point d'inflexion, qui correspond à la valeur maximum du coefficient $a$, et aux meilleures conditions d'utilisation du couple :

$$
a=\frac{-M_{0} \sqrt{2 A} \mathrm{e}^{-1 / 2}}{T_{\mathrm{c}}} \quad b=M_{0} \mathrm{e}^{-1 / 2}(\sqrt{2 A}+2)
$$

où $T_{\mathrm{c}}$ est la température du condenseur. On définit la température limite de désorption $\stackrel{\circ}{T}$ par :

$$
m(\stackrel{\circ}{T})=0 \quad \stackrel{\circ}{T}=\frac{b}{|a|}=\frac{T_{\mathrm{c}}(\sqrt{2 A}+2)}{\sqrt{2 A}} .
$$

Lorsque la température seuil, à laquelle débute la désorption, devient égale à $\stackrel{\circ}{T}$, on atteint la température limite d'évaporation $\mathrm{F}_{\mathrm{ev}}$ :

$$
\begin{gathered}
T_{\text {seuil }}=\frac{T_{\mathrm{c}}}{T_{\mathrm{ev}}}\left(T_{\text {nuit }}-T_{\mathrm{ev}}\right)+T_{\mathrm{c}} \\
\mathrm{F}_{\mathrm{ev}}=\frac{T_{\mathrm{c}} T_{\text {nuit }}}{\stackrel{\circ}{T}} .
\end{gathered}
$$

Dans le cas où $\stackrel{\circ}{T}$ est suffisamment élevée (cas de la zéolithe), le phénomène limitant la température d'évaporation est alors le système de captation :

$$
\mathrm{F}_{\mathrm{ev}}=\frac{T_{\mathrm{c}} T_{\text {nuit }}}{\mathrm{F}}
$$

où $\mathrm{F}$ est la température du capteur pour laquelle le rendement du capteur est nul à midi solaire.

Les calculs qui suivent sont faits pour un $\mathrm{kg}$ d'adsorbant. On appelle $T_{\mathrm{a}}$ la valeur moyenne de la température ambiante, servant de référence pour le calcul de l'exergie, et $T_{\max }$ la température maximum atteinte dans le capteur.

\subsection{CalCUl du RENDEMENT EXeRGÉTIQUe du SYSTÈme.} - $\mathrm{Si}$ on reporte la chaleur du chauffage isostérique de l'eau adsorbée dans le bilan des chaleurs sensibles, l'exergie nécessaire aux cycles s'écrit :

$$
E X . \text { nec. }=\sum_{i=0}^{N} \frac{\Delta H_{\mathrm{ads}}}{T_{i}}\left(T_{i}-T_{\mathrm{a}}\right) \overline{\Delta m}
$$

où $i$ est l'indice du cycle, et $\Delta H_{\text {ads }}$ l'enthalpie massique d'adsorption supposée constante dans les conditions du fonctionnement.

(La variation de $\Delta H_{\text {ads }}$ est en fait de l'ordre de $5 \%$ )

$$
N \overline{\Delta m}=m\left(T_{\text {seuil }}\right)-m\left(T_{\max }\right)=a\left(T_{\text {seuil }}-T_{\max }\right)
$$

de même

$$
i \overline{\Delta m}=a\left(T_{\text {seuil }}-T_{i}\right) \quad \text { et } \quad T_{i}=T_{\text {seuil }}-i \frac{\overline{\Delta m}}{a} .
$$


En supposant un grand nombre de cycles

$$
\begin{aligned}
& E X . \text { nec. }=N \overline{\Delta m} \Delta H_{\text {ads }}-\Delta H_{\text {ads }} T_{\mathrm{a}} \overline{\Delta m} \int_{x=0}^{N} \frac{\mathrm{d} x}{T_{\text {seuil }}-x \frac{\overline{\Delta m}}{a}} \\
& E X . \text { nec. }=a \Delta H_{\text {ads }}\left[\left(T_{\text {seuil }}-T_{\text {max }}\right)+T_{\mathrm{a}} \log \frac{T_{\text {max }}}{T_{\text {seuil }}}\right] .
\end{aligned}
$$

L'exergie désirée à l'évaporateur vaut : $E X$. des. $=N \overline{\Delta m} \frac{\Delta H_{\mathrm{ev}}}{T_{\mathrm{ev}}}\left(T_{\mathrm{a}}-T_{\mathrm{ev}}\right)$ où $\Delta H_{\mathrm{ev}}$ est l'enthalpie massique d'évaporation supposée constante. La deuxième approximation consiste à arrêter le développement du logarithme au second ordre (précision de l'ordre de $10 \%$ )

$$
\log \frac{T_{\max }}{T_{\text {seuil }}} \simeq \frac{T_{\text {max }}-T_{\text {seuil }}}{T_{\text {seuil }}}-\frac{\left(T_{\text {max }}-T_{\text {seuil }}\right)^{2}}{2 T_{\text {seuil }}^{2}}+\cdots
$$

On trouve

$$
\frac{1}{\eta_{\mathrm{EX}}^{\text {cycle }}}=\frac{E X . \text { nec. }}{E X . \text { des. }}=\frac{\Delta H_{\mathrm{ads}}}{\Delta H_{\mathrm{ev}}} \frac{T_{\mathrm{ev}}}{T_{\text {seuil }}\left(T_{\mathrm{a}}-T_{\mathrm{ev}}\right)}\left[T_{\text {seuil }}-\frac{3}{2} T_{\mathrm{a}}+\frac{T_{\mathrm{a}} T_{\mathrm{max}}}{2 T_{\text {seuil }}}\right]
$$

L'exergie nécessaire sous forme de chaleur sensible s'écrit :

$$
E X . \text { nec. }=\int_{T_{\mathrm{a}}}^{T_{\max }} \mathrm{d} E X=\int \mathrm{d} q\left(1-\frac{T_{\mathrm{a}}}{T}\right)=\int_{T_{\mathrm{a}}}^{T_{\max }} C^{\prime} \mathrm{d} T\left(1-\frac{T_{\mathrm{a}}}{T}\right)
$$

avec

$$
C^{\prime}=C_{\mathrm{p}}^{\text {adsorbant }}+C_{\mathrm{p}}^{\text {adsorbat }} m\left(T_{\text {seuil }}\right)+C_{\mathrm{p}}^{\text {eau }} \mu
$$

où $\mu$, valeur en eau du bâti par kg d'adsorbant, est supposée indépendante du choix du couple et des dimensions optima du capteur.

$$
\frac{1}{\eta_{\mathrm{EX}}^{\text {ch.sens. }}}=\frac{T_{\mathrm{c}} T_{\mathrm{ev}} C^{\prime}}{2 \Delta H_{\mathrm{ev}} T_{\mathrm{a}} M_{0} \sqrt{2 A} \mathrm{e}^{-1 / 2}\left(T_{\mathrm{a}}-T_{\mathrm{ev}}\right)} \frac{\left(T_{\max }-T_{\mathrm{a}}\right)^{2}}{\left(T_{\max }-T_{\text {seuil }}\right)}
$$

et

$$
\frac{1}{\eta_{\mathrm{EX}}^{\text {syst }}}=\frac{1}{\eta_{\mathrm{EX}}^{\text {cyle }}}+\frac{1}{\eta_{\mathrm{EX}}^{\text {ch.sens. }}}=D \frac{\left(T_{\max }-T_{\mathrm{a}}\right)^{2}}{\left(T_{\max }-T_{\text {seuil }}\right)}+B T_{\max }+C
$$

avec

$$
\begin{aligned}
D & =\frac{T_{\mathrm{c}} T_{\mathrm{ev}} C^{\prime}}{2 \Delta H_{\mathrm{ev}} T_{\mathrm{a}} M_{0} \sqrt{2 A} \mathrm{e}^{-1 / 2}\left(T_{\mathrm{a}}-T_{\mathrm{ev}}\right)} \quad B=\frac{\Delta H_{\mathrm{ads}} T_{\mathrm{ev}} T_{\mathrm{a}}}{2 \Delta H_{\mathrm{ev}} T_{\text {seuil }}^{2}\left(T_{\mathrm{a}}-T_{\mathrm{ev}}\right)} \\
C & =\frac{\Delta H_{\mathrm{ads}} T_{\mathrm{ev}}\left(T_{\text {seuil }}-3 / 2 T_{\mathrm{a}}\right)}{\Delta H_{\mathrm{ev}} T_{\text {seuil }}\left(T_{\mathrm{a}}-T_{\mathrm{ev}}\right)} .
\end{aligned}
$$

\subsection{RENDEMENT OPTIMUM DU SYSTÈME. -}

$\frac{1}{\eta_{\mathrm{EX}}^{\text {syst }}}$ admet un minimum pour $\frac{\mathrm{d}\left(\frac{1}{\eta_{\mathrm{EX}}^{\text {syst }}}\right)}{\mathrm{d} T_{\max }}=0$. Soit pour la température maximum atteinte dans le capteur qui optimise le rendement exergétique du système $\left(T_{\mathrm{opt}}^{\mathrm{sys}}\right)$

$$
T_{\text {opt }}^{\text {syst }}=T_{\text {seuil }}+\sqrt{\delta}
$$


où

$$
\begin{gathered}
\delta=T_{\text {seuil }}^{2}+\frac{D T_{\mathrm{a}}^{2}-2 D T_{\mathrm{a}} T_{\text {seuil }}-B T_{\text {seuil }}^{2}}{B+D} \\
\frac{1}{\eta_{\mathrm{EX}}^{\text {syst }}}(\text { minimum })=\frac{D\left(\sqrt{\delta}+T_{\text {seuil }}-T_{\mathrm{a}}\right)^{2}}{\sqrt{\delta}}+B\left(T_{\text {seuil }}+\sqrt{\delta}\right)+C .
\end{gathered}
$$

L'équation (9) décrit, dans le cadre des approximations considérées, l'ensemble des influences qui s'exercent sur le rendement optimum du système pour $T_{\text {opt }}^{\text {syst }}<\stackrel{\circ}{T}$.

Dans le cas où $T_{\mathrm{opt}}^{\text {syst }}>T$, la formule (9) se réécrit :

$$
\frac{1}{\eta_{\mathrm{EX}}^{\text {syst }}}=\frac{\Delta H_{\mathrm{ads}}\left(T_{\text {seuil }}-\stackrel{\circ}{T}+T_{\mathrm{a}} \log \frac{\stackrel{\circ}{T}}{T_{\text {seuil }}}\right) T_{\mathrm{ev}}}{\Delta H_{\mathrm{ev}}\left(T_{\text {seuil }}-\stackrel{\circ}{T}\right)\left(T_{\mathrm{a}}-T_{\text {ev }}\right)}+\frac{C^{\prime}\left(\stackrel{\circ}{T}-T_{\mathrm{a}}-T_{\mathrm{a}} \log \frac{\stackrel{\circ}{T}}{T_{\mathrm{a}}}\right) T_{\mathrm{ev}} T_{\mathrm{c}}}{\Delta H_{\mathrm{ev}} M_{0} \sqrt{2 A} \mathrm{e}^{-1 / 2}\left(\stackrel{\circ}{T}-T_{\text {seuil }}\right)\left(T_{\mathrm{a}}-T_{\text {ev }}\right)} .
$$

4. 3 Etude de L'influence $T_{\mathrm{c}}$ ET $T_{\mathrm{ev}}$ POUR Les deuX COUPLES : ZÉOLITHE 13X-EAU ET GEL DE SILICE-EAU. Les données concernant ces couples sont tirées des notices techniques fournies par deux fabricants $\left({ }^{1}\right)$ :

$$
\text { zéolithe-eau : } \begin{aligned}
\Delta H_{\mathrm{ev}} & =2,490 \times 10^{6} \mathrm{~J} / \mathrm{kg} \\
\Delta H_{\mathrm{ads}} & =3,250 \times 10^{6} \mathrm{~J} / \mathrm{kg} \\
M_{0} & =0,269 \mathrm{~kg} / \mathrm{kg} \text { zéolithe anhydre } \\
A & =4,33 \mathrm{S.I} . \\
C^{\prime} & =2300 \mathrm{~J} / \mathrm{degré}, \mathrm{kg} \\
\text { gel-eau : } \quad \Delta H_{\mathrm{ads}} & =3,018 \times 10^{6} \mathrm{~J} / \mathrm{kg} \\
M_{0} & =0,40 \mathrm{~kg} / \mathrm{kg} \\
A & =100 \mathrm{S.I} \\
C^{\prime} & =1830 \mathrm{~J} / \text { degré, } \mathrm{kg} .
\end{aligned}
$$

(voir tableau).

4.4 Discussion. - Certaines des approximations introduites dans le calcul tendent à majorer assez nettement le $\eta_{\mathrm{EX}}^{\text {syst }}$ (optimum), (de l'ordre de $20 \%$ ), et donc à donner une valeur de $T_{\mathrm{opt}}^{\text {syst }}$ au-dessous de la réalité $\left(10\right.$ à $\left.15^{\circ} \mathrm{C}\right)$. La formule $(9)$ apparaît cependant suffisamment significative pour établir une comparaison permettant le choix de l'appareil optimisé, dans la plupart des cas.

La comparaison sur le tableau des couples zéolithe 13X-eau et gel de silice-eau, fait apparaître qu'audessus de $-5^{\circ} \mathrm{C}$ à l'évaporateur, le gel est nettement plus performant (environ deux fois), quelle que soit la température de condensation. Au-dessous de $-\tilde{5}^{\circ} \mathrm{C}$ le gel devient inutilisable, ayant atteint sa température limite d'évaporation, cependant que la zéolithe continue à avoir une assez bonne efficacité (baisse de $25 \%$ du C.O.P. entre $0^{\circ} \mathrm{C}$ et $-15^{\circ} \mathrm{C}$ ), mais nécessite un type de capteur de très bonne qualité (sous-vide et à surface sélective). Le type de capteur

( $\left.{ }^{1}\right)$ Davidson. - Molecular Sieves Ind. Chem. Dept., Baltimore, Maryland. CECA, S.A., 14600 Honfleur. adapté au gel est moins performant que celui de la zéolithe dans tous les modes d'utilisation (condenseur à air ou à eau). Il en résulte pour le C.O.P. global une amélioration pour la zéolithe dans le cas d'utilisation de capteur à haute performance (par suite de la meilleure adaptation du capteur et donc de la valeur plus grande du $\left.\eta_{\mathrm{EX}}^{\text {capt }}\right)$. Cet avantage ne semble être déterminant que dans le domaine $\left(-5^{\circ} \mathrm{C}, 0^{\circ} \mathrm{C}\right)$ à l'évaporateur, avec un condenseur à air et un capteur à surface sélective sous-vide.

En conclusion, le gel de silice est nettement plus performant que la zéolithe dans le domaine de température d'utilisation du froid supérieur à $0^{\circ} \mathrm{C}$ (petite réfrigération domestique, conservation des vaccins, conservation des denrées agricoles en région tropicale). On peut employer des condenseurs à convection naturelle d'air donnant une totale autonomie d'utilisation, et les capteurs les mieux adaptés sont les capteurs plans à surface sélective, dont la technologie bien développée, limite les coûts de revient. On pourrait alors atteindre un C.O.P. global de 0,30 .

Signalons qu'en dessous de $0^{\circ} \mathrm{C}$, le froid est produit par sublimation de la glace dans l'évaporateur; il en résulte un abaissement important du coefficient d'échange thermique à la surface de l'évaporateur par suite d'une part, de l'absence de convection du fluide, du mauvais coefficient de transmission de la chaleur dans la glace, et de la surface de contact réduite entre la glace et l'évaporateur. Cela a pour conséquence un retard dans le phénomène de réadsorption et la nécessité de surdimensionner l'évaporateur.

\section{Conclusions.}

Le calcul analytique qui a été fait a permis de trouver une formule qui donne les performances théoriques à attendre d'un réfrigérateur solaire à adsorption solide, lorsqu'on utilise différents couples d'adsorption et pour une grande variété de conditions d'uti- 


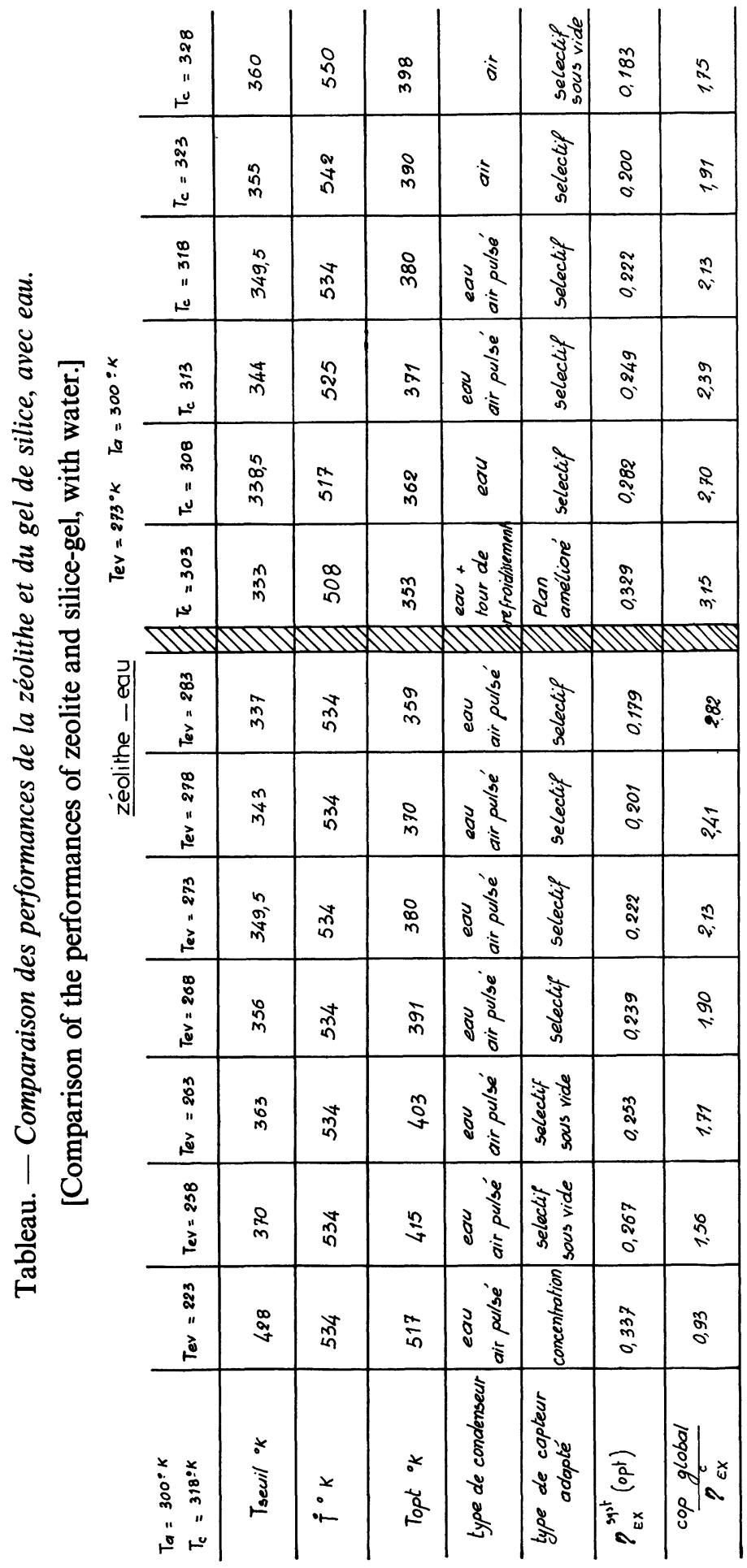

\begin{tabular}{|c|c|c|c|c|c|c|c|}
\hline$\stackrel{\infty}{\stackrel{\infty}{m}}$ & : & $\stackrel{n}{n}_{n}^{n}$ & $\begin{array}{c}0 \\
\text { co } \\
\text { m }\end{array}$ & ริ & $\begin{array}{l}\text { ปูँ } \\
\text { ปั }\end{array}$ & $\begin{array}{l}\infty \\
\stackrel{n}{n} \\
0 \\
0\end{array}$ & $\underset{\text { m. }}{\stackrel{n}{+}}$ \\
\hline$\stackrel{m}{\tilde{m}}$ & 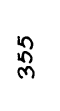 & के & $\stackrel{R}{m}$ & "亏 & $\begin{array}{l}\text { डुँ } \\
\text { ปूँ }\end{array}$ & $\begin{array}{c}\stackrel{\circ}{0} \\
0 \\
0\end{array}$ & $\begin{array}{l}\infty \\
\infty \\
m \\
m\end{array}$ \\
\hline$\stackrel{\infty}{m}$ & م్ & $\begin{array}{l}\stackrel{n}{0} \\
\stackrel{m}{n}\end{array}$ & $\begin{array}{l}m \\
\text { ò } \\
m\end{array}$ & 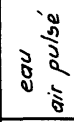 & $\begin{array}{l}\text { ఫूँ } \\
\text { ऽँ }\end{array}$ & 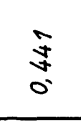 & $\underset{\forall}{\mathbb{N}}$ \\
\hline$\frac{n}{n}$ & 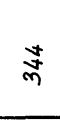 & $\begin{array}{l}\hat{n} \\
n\end{array}$ & $\begin{array}{l}n \\
\hat{n} \\
m\end{array}$ & 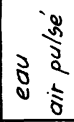 & 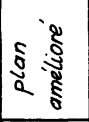 & \begin{tabular}{l}
$\infty$ \\
\hdashline \\
\hdashline
\end{tabular} & $\underset{8}{9}$ \\
\hline $\begin{array}{l}\infty \\
0 \\
m \\
m\end{array}$ & 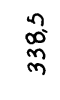 & స్ & $\begin{array}{c}\infty \\
\text { m }\end{array}$ & รู้ & 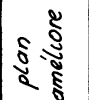 & $\begin{array}{l}\text { nn } \\
\text { on } \\
0\end{array}$ & $\begin{array}{l}\stackrel{\infty}{D} \\
\stackrel{n}{n}\end{array}$ \\
\hline $\begin{array}{c}\text { ồ } \\
11 \\
\omega\end{array}$ & m & \) & స్ & $\begin{array}{l}++ \\
8 \\
8 \\
8\end{array}$ & $\S$ & 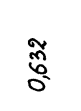 & $\underset{8}{8}$ \\
\hline$\pi$ & $\Delta$ & & 1 & & 11 & $M$ & $\Delta$ \\
\hline$\stackrel{m}{\infty}$ & 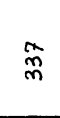 & $\stackrel{m}{m}$ & 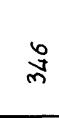 & 3. & §ૂ & 商 & 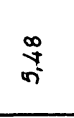 \\
\hline$\stackrel{\infty}{\infty}$ & ले & ळ్ & 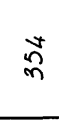 & 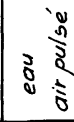 & ईह & \begin{tabular}{l}
8 \\
\multirow{8}{8}{} \\
0
\end{tabular} & $\begin{array}{l}8 \\
\mathbb{5} \\
v^{2}\end{array}$ \\
\hline$\stackrel{m}{a}$ & 怘 & $\stackrel{m}{m}$ & ڤ̊ & 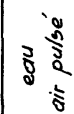 & $\begin{array}{l}\text { जे } \\
\text { ूँ๊ } \\
\text { जั }\end{array}$ & 疍 & 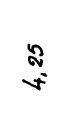 \\
\hline $\begin{array}{l}\infty \\
\stackrel{D}{2}\end{array}$ & 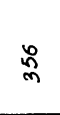 & $\begin{array}{l}n \\
\text { Dे } \\
m\end{array}$ & iे & 瓷 & 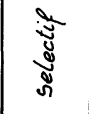 & 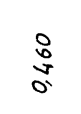 & 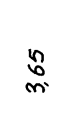 \\
\hline$\stackrel{n}{0}$ & $\stackrel{m}{\stackrel{m}{\infty}}$ & ڤొ & $\stackrel{\infty}{\infty}$ & 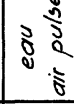 & & 0 & 0 \\
\hline $\begin{array}{l}\infty \\
2 \\
2\end{array}$ & 足 & 吕 & 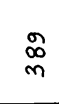 & 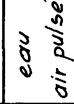 & & 0 & 0 \\
\hline 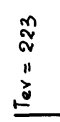 & $\stackrel{\infty}{\stackrel{\infty}{*}}$ & $\begin{array}{l}n \\
\stackrel{n}{n}\end{array}$ & के & 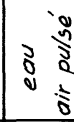 & & 0 & 0 \\
\hline $\begin{array}{cc} \\
0 \\
0 \\
0\end{array}$ & ฐ్ & or & $\begin{array}{l}{ }^{*} \\
\stackrel{a}{\circ}\end{array}$ & 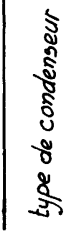 & 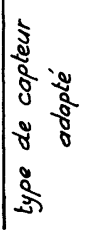 & $\frac{\widehat{a}}{\sqrt[0]{0}}$ & 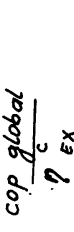 \\
\hline
\end{tabular}


lisation. Il devrait permettre d'inventorier les possibilités d'utilisation de nouveaux couples et en particulier les possibilités d'utilisation des réfrigérants traditionnels (fréons, $\mathrm{NH}_{3}$ alcools) dont beaucoup sont adsorbables par les tamis moléculaires.

Le manque de précision des résultats obtenus (précision de l'ordre de $20 \%$ ), dû à certaines hypothèses rendues nécessaires pour la simplicité du calcul, fait que dans un certain nombre de cas, il ne sera pas possible de faire le choix du meilleur appareil (rendement voisin, non-adaptation couple-capteur). Il faut alors utiliser une méthode plus précise, telle qu'une modélisation numérique à l'ordinateur [7], où la méthode graphique de la référence [6].

La comparaison qui a été faite entre les couples zéolithe $13 x$-eau et gel de silice ultramicroporeux-eau. a montré le net avantage de ce dernier couple dans le domaine d'utilisation du froid qui correspond en particulier à la conservation en entrepôt des produits vivriers d'origine tropicale (température supérieure à $8^{\circ} \mathrm{C}$ ), ce qui permettrait d'envisager une solution nouvelle à cet important problème des pays chauds en développement [8]. Un projet en cours vise à la réalisation d'un tel appareil.

\section{Bibliographie}

[1] Meunier, F., Cahier AFEDES 5 (1978).

[2] TChernev, D. I., Proceedings of the XIVth Intersociety Energy Conversion Engineering Conferences (1979).

[3] Guilleminot, J. J., Meunier, F., Rev. Gen. Therm. 239 (1981).

Dupont, M., Meunier, F., Nguyen, P. H., Second National Conference on renewable energy PortoRico (1982).

ADELl, A., à paraître.
[4] MaczeK, K., GutKowski, K. M., Application of various criteria for optimizing refrigerating plant. In Saving of Energy in refrigeration I.I.F. (1980).

[5] Vokaer, D., Bougard, J., Proceedings of the ISES Congress Brighton (1981).

[6] Adell, A., Proceedings of the XVIth International Refrigeration Congress Paris (1983).

[7] Guilleminot, J. J., Meunier, F., Mischler, B., Revue Phys. appl. 15 (1980).

[8] Adell, A., Rev. Gen. Froid (1982). 